\title{
HIBRID APPROACH OF CAMERAS AND GPS FOR DISPLACEMENT MEASUREMENTS OF SUPER LONG-SAPN BRIDGES
}

\author{
Wonjo Jung ${ }^{1}$, Do Hyoung Shin ${ }^{2 *}$, SungKwon Woo ${ }^{2}$, Won-jin Park ${ }^{2}$, and Sangbin Kim ${ }^{2}$ \\ ${ }^{1}$ Virtual Globe Team, SK C\&C, Seongnam, Korea \\ ${ }^{2}$ Department of Civil Engineering, Inha University, Incheon, Korea \\ *Corresponding author (dshin@inha.ac.kr)
}

\begin{abstract}
In a recent decade, the GPS has been introduced to directly measure the displacements of super long-span bridges. However, due to its high cost, it is not appropriate to apply a number of GPS receivers in a super-long span bridge in order to measure the displacements through the overall bridge. From the limited point-based measurements of the displacement, a mode shape model for the bridge maintenance gets more complicated and includes errors due to the assumption in the displacements other than the measured points. This study considers digital cameras as the supplementary approach for the displacement measurements to address the limitation of GPS receivers. The objective of this study is to theoretically investigate the feasibility to use digital cameras with GPS receivers to develop a more compelling mode shape model for the super long-span bridge maintenance. From a case study, it was found that digital cameras can provide a sufficient measuring width with a moderate resolution to measure the displacements of a super long-span bridge.
\end{abstract}

Keywords: Super Long-span Bridge, Displacement, Digital Camera, GPS, Maintenance

\section{INTRODUCTION}

In South Korea, the facility maintenance systems (FMSs) have been applied in the bridge since the mid 1990s when the Dolsan Bridge and the Jindo Bridge employed them. Particularly for the super long-span bridges such as the Seohae Bridge, the Youngjong Bridge, and the Incheon Bridge, the FMSs have been well developed and adopted.

However, most of the FMSs for the super long-span bridges have been based on the estimations of the displacements from the measurements of the accelerations or strains of the bridges. These indirect approaches to estimate the displacements were generally used due to the difficulty to directly measure the displacements of the super long-span bridges. Even though it is relatively easy and inexpensive to install accelerometers or strain gages as the indirect approaches, the complicated calculations are required and the errors can be accumulated while estimating the displacements from their measurements. Thus, the indirect estimations of the displacements from the measurements of the accelerations or strains are less reliable than the direct measurements of the displacements.
To address this issue, recently GPS receivers have been employed to directly measure of the displacements of the super long-span bridges. However, a limited number of GSP receivers are usually used in a bridge due to its high costs, thus providing the displacement measurements of the only specific points where GPS receivers are located.

The research project "Structural Health Monitoring Based on Displacement Measurement" funded by MLTM which the authors are involved in aims to develop a new approach to estimate the structural status such as stresses, strains or accelerations of an overall bridge from the displacement measurements with GPS receivers. For the estimations of the comprehensive structural status, the displacement measurements though the overall bridge are required. However, as mentioned above, a limited number of GPS receivers cannot provide the displacement measurements through an overall bridge.

To address this lack of the displacement measurements with GPS receivers, a mode shape model for super longspan bridges will be developed in the research project "Structural Health Monitoring Based on Displacement 
Measurement". Through the model, the displacements of the points where GPS receivers are not located will be estimated. The structural status of a bridge can be also estimated for the maintenance from the model. The model would be modified with the comparison between the measured displacements from the GPS receivers and the estimated displacements from the model, and finally determined when the differences are small enough.

However, the model probably would not be accurate if the comparisons are made with a limited number of the measurement points with GPS receivers. This study was conducted as a part of the research project "Structural Health Monitoring Based on Displacement Measurement" to find out the solutions to address this limitation. This study considers digital cameras as the supplementary approach for the displacement measurements. The objective of this study is to theoretically investigate the feasibility to employ digital cameras with GPS receivers to develop a more compelling mode shape model for the super long-span bridge maintenance.

\section{RELATED TECHNOLOGIES}

There are some approaches to directly measure the displacements of super long-span bridges. The first approach is a laser displacement sensor. While laser displacement sensors provide a high accuracy in the displacement measurements, they are susceptible to the weather such as frog. For a high accuracy, laser displacement sensors require a laser receiver and a transmitter to be located each other within approximately $400 \mathrm{~m}$, thus providing a relatively short measuring distance. The measuring resolution also proportionally decreases as the measuring distance increases. Its high cost is another drawback.

The second approach is a GPS receiver. GPS receivers overcome the susceptibility to the weather of laser displacement sensors while they also provide a high accuracy in the displacement measurements if DGPS is employed. Bae and Ha (2008) presents that the GPS receivers provide the similar measurements of the displacements of the bridges with those with the laser displacement sensors. Recently, GPS receivers are widely used to measure the displacements of super long-span bridges despite their high costs. However, due to the high costs, a limited number of GPS receivers are usually installed in a bridge. For example, the Youngjong Bridge and the Incheon have only 8 and 6 GPS receivers in their main spans, respectively.

The third approach is a digital camera. While this approach is still in the early stage, it has a potential benefit in terms of a cost. One of its critical drawbacks is that digital cameras are susceptible to the weather as laser displacement sensors are. Another drawback is that a digital camera and a target should be located relatively closely each other. Otherwise, the measuring resolution would be deteriorated. Jeong et al. (2005) demonstrated that digital cameras probably can replace with laser displacement sensors or GPS receivers in terms of the accuracy in the displacement measurements. Their system worked in a similar manner to laser displacement sensors. The difference between a laser displacement sensor and the digital camera system of Jeong and his colleagues is that the one uses an active laser target while the other uses a passive target that is a printed mark. On the other hand, in the both approaches, a laser receiver or digital camera has a very limited view around a target. That is, the digital camera system of Jeong and his colleagues, as laser displacement sensors do, can measure the displacements of only specific points where targets are located.

While this study also considers digital cameras to measure the displacements of a super long-span bridge, we have a different view in exploiting the benefits of digital cameras from one of Jeong et al. (2005). The main concern of their study was to explore digital cameras as the replacement of expensive GPS receivers or laser displacement sensors. In this study, however, digital cameras are not considered as the replacement of GPS receivers or laser displacement sensors. Instead, this study explores digital cameras as the supplementary method for the displacement measurements of a super long-span bridge.

\section{MEASURING SCHEMATIC WITH DIGITAL CAMERAS}


Laser displacement sensors or digital cameras are susceptible to the weather, which is a critical defect for the stable measurements of the displacements of super longspan bridges. Therefore, we have determined that the primary approach for the displacement measurements in the research project "Structural Health Monitoring Based on Displacement Measurement" is GPS receivers that provide stable and reliable measurements regardless of the weather.

As explained above, however, GPS receivers provide the displacement measurements of only specific points where they are located. This study considers digital cameras as a secondary approach for the displacement measurements to supplement the lack of points with GPS receivers. From the displacement measurements of more points of a bridge, a more compelling mode shape model, from which the displacements and the structural status of an overall bridge would be estimated, can be developed.

The main concerns in employing digital cameras as a supplementary method are to (1) measure the displacements of as many points of a bridge as possible while (2) obtaining a high accuracy and resolution. The first concern would be achieved more easily if each camera can cover a more area along the spans of a bridge in its field of view. Therefore, this study considers each digital camera to provide the line-based measurements for the displacements instead of the point-based measurements which GPS receivers produce.

However, the large field of view by zooming in a scene causes a loss in the image resolution. A focal length decreases or increases as a zoom-in or a zoom-out are made, respectively. As a focal length decreases, the image of a larger view is projected on an image sensor. On the other hand, as a focal length increases, the image of a smaller view is projected on an image sensor.

The image sensors such as CCD or CMOS sensors of digital cameras consist of millions of pixels (cells). When the image of a view is projected on the image sensor, the sensor splits the image into millions of pixels. As the image of a larger view is projected on an image sensor, the area of the view which each pixel of the sensor captures increases. For example, if the image of a $6 \mathrm{~m}$ (width) $\mathrm{x} 4 \mathrm{~m}$ (height) view is projected on the image sensor consisting of 600 (width) x 400 (height) pixels each of which is $6.4 \mu \mathrm{m}$ square, the area the view captured by each pixel is $1 \mathrm{~cm}$ (width) $\times 1 \mathrm{~cm}$ (height). If the image of a $3 \mathrm{~m}$ (width) x 2 $\mathrm{m}$ (height) view is projected on the same sensor, the area of the view captured by each pixel is $0.5 \mathrm{~cm}$ (width) $\mathrm{x} 0.5 \mathrm{~cm}$ (height). That is, as the field of view increases, the image resolution decreases.

The main concern of this study is focused on obtaining a large field of view with a high image resolution. Therefore, this study theoretically investigates the field of views acquired with specific resolutions in order to explore the feasibility to employ digital cameras as the supplementary method for the displacement measurements of a super long-span bridge.

Besides the field of view and the image resolution, the measurement accuracy is significantly important for the compelling measurements of the displacements. Even if digital cameras provide the images with high resolutions, only relative displacements can be precisely measured from the images. One of the approaches to address this accuracy issue is to refer to the displacement measurements from GPS receivers. If the image of a bridge includes the points where the GPS receivers are located, it is possible to create the matrix for mapping the relative displacements in the pixel coordinates to the displacements in the global coordinates based on those of the points with the GPS receivers. The issue regarding to the measurement accuracy is out of the scope of this study, and the details will be discussed in another study later.

In the following section, the theoretical investigations on the rates of the field of views and the image resolutions based on a case study are discussed.

\section{CASE STUDY}

This study calculated the maximum length along the spans that can be captured by each camera when the required measuring resolutions are $1 \mathrm{~mm}$ or $5 \mathrm{~mm}$ from a case study. For the digital camera for the case study, the EOS 5D Mark II camera from Canon was selected because it provides a high pixel resolution (5616 width x 3744 height) with a moderate cost. 
Figure 1 illustrates the relationship between the required resolutions and the pixel size. The ratio of the pixel size (= $6.4 \mu \mathrm{m})$ of the EOS 5D Mark II camera to the required resolution $(1 \mathrm{~mm}$ or $5 \mathrm{~mm})$ is equal to that of the focal length to the measuring distance. Therefore,

$$
\mathrm{D}=\frac{\text { Required resolution }}{6.4 \mu \mathrm{m}} \mathrm{f}
$$

Thus, for the required resolution of $5 \mathrm{~mm}, \mathrm{D}=781.25 \cdot \mathrm{f}$ (m). For the required resolution of $1 \mathrm{~mm}, \mathrm{D}=156.25 \cdot \mathrm{f}(\mathrm{m})$.

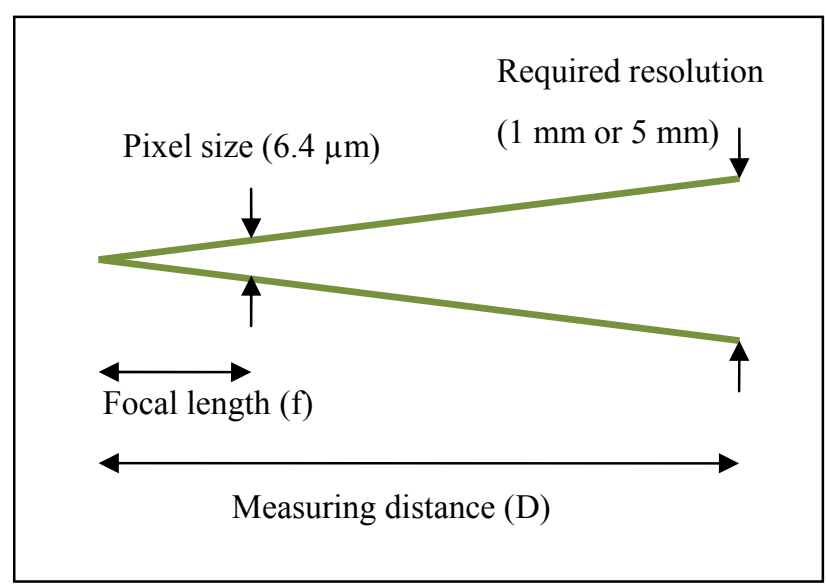

Fig. 1 Required resolutions and pixel size

If the view direction of the camera is horizontally perpendicular to the spans, the maximum length along the spans (maximum measuring width) which can be captured by the camera is $28.08 \mathrm{~m} \mathrm{(=5} \mathrm{mm} \times 5616)$ when the required resolution is $5 \mathrm{~mm}$, and $5.62 \mathrm{~m} \mathrm{(=1} \mathrm{mm} \times 5616)$ when the required resolution is $1 \mathrm{~mm}$.

If the view direction of the camera is vertically perpendicular to the spans, the maximum height of the span view (maximum measuring height) which can be captured by the camera is $18.72 \mathrm{~m}(=5 \mathrm{~mm} \times 3744)$ when the required resolution is $5 \mathrm{~mm}$, and $3.74 \mathrm{~m}(=1 \mathrm{~mm} \mathrm{x}$ 3744) when the required resolution is $1 \mathrm{~mm}$.

Table 1 shows the maximum measuring distances (D) according to the focal lengths to satisfy the required resolutions of $5 \mathrm{~mm}$ and $1 \mathrm{~mm}$ when the view direction of the camera is horizontally and vertically perpendicular to the spans. The maximum measuring widths (W) and heights $(\mathrm{H})$ captured by the camera at the maximum measuring distances are also presented in Table 1.
Table 1 Maximum measuring distances, widths, and heights according to the focal lengths at $\alpha=0^{\circ}$ and $\beta=0^{\circ}$

\begin{tabular}{lllllll} 
Focal & \multicolumn{2}{l}{ Req. resolution $=5 \mathrm{~mm}$} & \multicolumn{3}{c}{ Req. resolution $=1 \mathrm{~mm}$} \\
\cline { 2 - 7 } $\begin{array}{l}\text { length } \\
(\mathrm{mm})\end{array}$ & $\mathrm{Max}$ & $\mathrm{Max}$ & $\mathrm{Max}$ & $\mathrm{Max}$ & $\mathrm{Max}$ & $\mathrm{Max}$ \\
\hline 35 & 27.34 & 28.08 & 18.72 & 5.47 & 5.62 & 3.74 \\
\hline 50 & 39.06 & 28.08 & 18.72 & 7.81 & 5.62 & 3.74 \\
\hline 100 & 78.13 & 28.08 & 18.72 & 15.63 & 5.62 & 3.74 \\
\hline 300 & 234.38 & 28.08 & 18.72 & 46.88 & 5.62 & 3.74 \\
\hline 500 & 390.63 & 28.08 & 18.72 & 78.13 & 5.62 & 3.74 \\
\hline 800 & 625.00 & 28.08 & 18.72 & 125.0 & 5.62 & 3.74 \\
\hline
\end{tabular}

The measuring width and height vary with the angles at which the view direction of the camera is oblique to the spans horizontally and vertically. Figures 2 and 3 illustrate the view direction of the camera oblique to the spans horizontally at the angle of $\alpha$ and vertically at the angle of $\beta$, respectively, at the measuring distance of $\mathrm{D}$.

From Figure 2, the maximum measuring width (W) captured by a digital camera at $\mathrm{D}$ with the view direction of $\alpha$ and $\beta$ is derived as follows:

$$
\begin{aligned}
& \delta=\tan ^{-1}\left(\frac{\mathrm{x}_{\mathrm{h}}}{\mathrm{f}}\right), \delta^{\prime}=\tan ^{-1}\left(\frac{\mathrm{x}_{\mathrm{h}}-\text { pixel size }}{\mathrm{f}}\right) \\
& \mathrm{W}=\mathrm{y}_{\mathrm{h}}^{\prime}-\mathrm{y}_{\mathrm{h}}=\mathrm{D}_{\mathrm{x}} \tan (\alpha+\delta)-\mathrm{D}_{\mathrm{x}} \tan (\alpha-\delta) \\
& \mathrm{D}_{\mathrm{x}} \leq \frac{\text { Required Resolution }}{\tan (\alpha+\delta)-\tan \left(\alpha+\delta^{\prime}\right)}
\end{aligned}
$$

From Figure 3, the maximum measuring height $(\mathrm{H})$ captured by a digital camera at $\mathrm{D}$ with the view direction of $\alpha$ and $\beta$ is derived as follows:

$$
\begin{aligned}
& \gamma=\tan ^{-1}\left(\frac{\mathrm{x}_{\mathrm{v}}}{\mathrm{f}}\right), \gamma^{\prime}=\tan ^{-1}\left(\frac{\mathrm{x}_{\mathrm{v}}-\text { pixel size }}{\mathrm{f}}\right) \\
& \mathrm{H}=\mathrm{y}_{\mathrm{v}}^{\prime}-\mathrm{y}_{\mathrm{v}}=\frac{\mathrm{D}_{\mathrm{y}} \tan (\beta+\gamma)-\mathrm{D}_{\mathrm{y}} \tan (\beta-\gamma)}{\tan \beta} \\
& \mathrm{D}_{\mathrm{y}} \leq \frac{\text { Required Resolution }}{\tan (\beta+\gamma)-\tan \left(\beta+\gamma^{\prime}\right)}
\end{aligned}
$$

In practice, it is most likely that the view direction is oblique to the spans due to physical constraints of the points where digital cameras are located. For example, the spans of the Incheon Bridge are approximately $50 \mathrm{~m}$ over the surface of the water, and it is quite difficult to install digital cameras vertically perpendicular to the spans. 


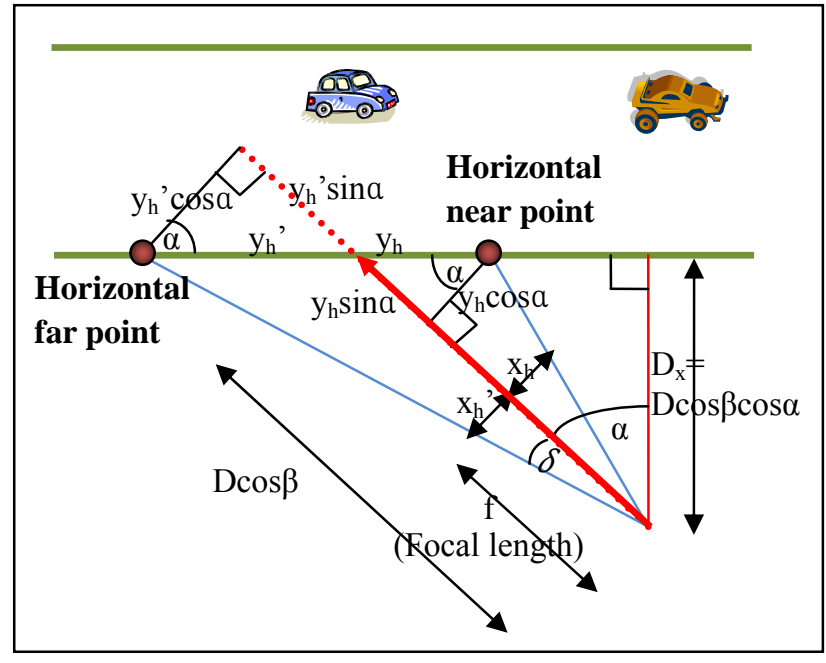

Fig. 2 View direction horizontally oblique to the spans

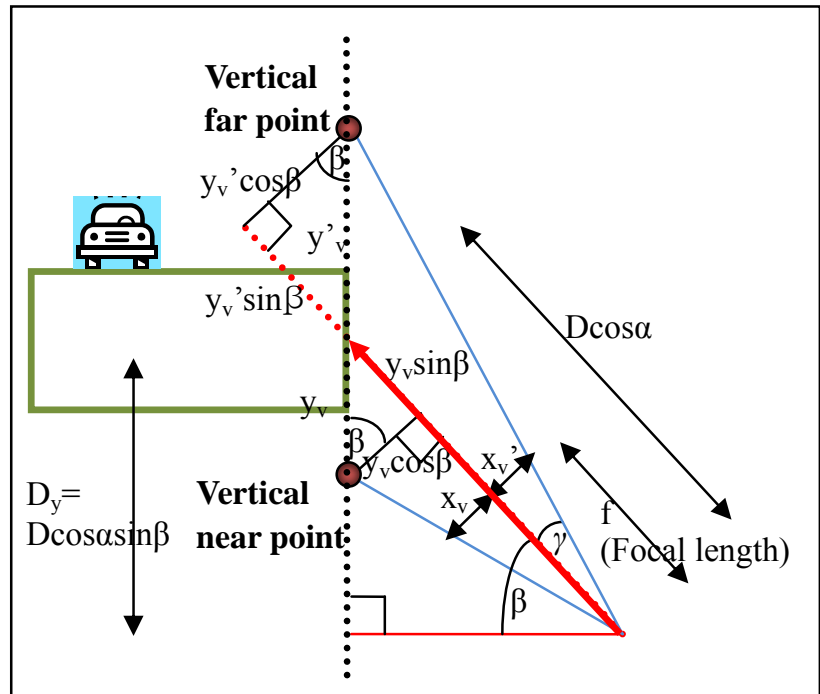

Fig. 3 View direction vertically oblique to the spans

Even though the number of the possible combinations of $\alpha$ and $\beta$ is infinite, the paper presents two cases (one at $\alpha=45^{\circ}$ and $\beta=45^{\circ}$ and the other at $\alpha=60^{\circ}$ and $\beta=60^{\circ}$ ) as the examples in order to see the maximum measuring widths and heights in those hard conditions. If the maximum measuring widths and heights are large enough to be used even in those tough conditions, this may indicate the feasibility of digital cameras as the supplementary approach to measure the displacements of a super longspan bridge.

Table 2 shows the maximum measuring distances (D), widths (W), and heights $(\mathrm{H})$ according to the focal lengths at $\alpha=45^{\circ}$ and $\beta=45^{\circ}$ when the required measuring resolution is $5 \mathrm{~mm}$ or $1 \mathrm{~mm}$. Table 3 shows those at $\alpha=60^{\circ}$ and $\beta=60^{\circ}$.

Table 2 Maximum measuring distances, widths, and heights according to the focal lengths at $\alpha=45^{\circ}$ and $\beta=45^{\circ}$

\begin{tabular}{lllllll}
\multirow{2}{*}{$\begin{array}{l}\text { Focal } \\
\text { length }\end{array}$} & \multicolumn{3}{l}{ Req. resolution $=5 \mathrm{~mm}$} & \multicolumn{3}{l}{ Req. resolution $=1 \mathrm{~mm}$} \\
\cline { 2 - 7 }$(\mathrm{mm})$ & $\mathrm{Max}$ & $\mathrm{Max}$ & $\mathrm{Max}$ & $\mathrm{Max}$ & $\mathrm{Max}$ & $\mathrm{Max}$ \\
\hline 35 & 7.46 & 9.02 & 9.18 & 1.49 & 1.80 & 1.84 \\
\hline 50 & 15.98 & 13.24 & 11.49 & 3.20 & 2.65 & 2.30 \\
\hline 100 & 47.91 & 19.55 & 14.73 & 9.58 & 3.91 & 2.95 \\
\hline 300 & 181.96 & 24.94 & 17.31 & 36.39 & 4.99 & 3.46 \\
\hline 500 & 317.02 & 26.17 & 17.87 & 63.40 & 5.23 & 3.57 \\
\hline 800 & 519.86 & 26.89 & 18.20 & 103.97 & 5.38 & 3.64 \\
\hline
\end{tabular}

Table 3 Maximum measuring distances, widths, and heights according to the focal lengths at $\alpha=60^{\circ}$ and $\beta=60^{\circ}$

\begin{tabular}{lllllll}
\hline \multirow{2}{*}{$\begin{array}{l}\text { Focal } \\
\text { length }\end{array}$} & \multicolumn{3}{l}{ Req. resolution $=5 \mathrm{~mm}$} & \multicolumn{3}{c}{ Req. resolution $=1 \mathrm{~mm}$} \\
\cline { 2 - 7 }$(\mathrm{mm})$ & $\mathrm{D}(\mathrm{m})$ & $\mathrm{Wax}(\mathrm{m})$ & $\mathrm{Max}$ & $\mathrm{Max}$ & $\mathrm{Max}$ & $\mathrm{Max}$ \\
\hline 35 & 1.96 & 1.63 & 4.78 & 0.39 & 0.33 & 0.96 \\
\hline 50 & 6.41 & 6.53 & 7.74 & 1.28 & 1.31 & 1.55 \\
\hline 100 & 28.16 & 14.76 & 12.30 & 5.63 & 2.95 & 2.46 \\
\hline 300 & 128.78 & 22.83 & 16.32 & 25.76 & 4.57 & 3.26 \\
\hline 500 & 231.58 & 24.82 & 17.25 & 46.32 & 4.96 & 3.45 \\
\hline 800 & 386.30 & 26.02 & 17.80 & 77.26 & 5.20 & 3.56 \\
\hline
\end{tabular}

As shown in Tables 2 and 3, the maximum measuring width and height decrease as the sizes of $\alpha$ and $\beta$ increase. However, it should be also noted that the decreases are not much significant for the large focal lengths. Especially for the focal length of $800 \mathrm{~mm}$, the maximum width for the required resolution of $5 \mathrm{~mm}$ decreases by only $6.8 \%$ and $0.6 \%$ when the obliqueness changes from $\alpha=0^{\circ}, \beta=0^{\circ}$ to $\alpha=45^{\circ}, \beta=45^{\circ}$ and when it changes from $\alpha=45^{\circ}, \beta=45^{\circ}$ to $\alpha=60^{\circ}, \beta=60^{\circ}$, respectively. The maximum height for that decreases by only $2.8 \%$ and $2.2 \%$, respectively. This finding indicates that even if digital cameras are oblique to the spans, the obliqueness would not much significantly deteriorate the maximum measuring widths and heights. 
For the displacement measurements of a super long-span bridge, a large measuring height is not required. For example, the maximum displacement of the Yongjong Bridge is approximately $35 \mathrm{~mm}$. If cameras have the view direction to the horizontal center line of side of the spans and the center line is marked, the required measuring height would be just a few centimeters around the center line. Therefore, the maximum measuring heights of $17.8 \mathrm{~m}$ or $3.56 \mathrm{~m}$ which are for the required resolutions of $5 \mathrm{~mm}$ or $1 \mathrm{~mm}$ respectively at $\alpha=60^{\circ}, \beta=60^{\circ}$ are allowable.

The maximum measuring width of $5.2 \mathrm{~m}$ which is for the required resolution of $1 \mathrm{~mm}$ at $\alpha=60^{\circ}, \beta=60^{\circ}$ is relatively small. For example, the length of the suspension part of the Youngjong Bridge is $550 \mathrm{~m}$ and the 8 GPS receivers are located in the spans with the intervals of approximately $100-200 \mathrm{~m}$. If each camera covers $5.2 \mathrm{~m}$ around one of the 8 points with the GPS receivers, approximately $83.2 \mathrm{~m}(=5.2 \times 16)$ can be covered by the 16 digital cameras. The area measured by the digital cameras would be only $15 \%$ of the suspension part. If the required resolution of $5 \mathrm{~mm}$ at $\alpha=60^{\circ}, \beta=60^{\circ}$ is considered, the maximum measuring width is $26.02 \mathrm{~m}$ and approximately $416.3 \mathrm{~m}(=26.02 \times 16)$ can be covered by the 16 digital cameras. That is, $76 \%$ of the suspension part would be measured by the digital cameras.

In the consideration that the price of the EOS 5D Mark II camera is about $\$ 3,500$, it does not seem feasible to spend $\$ 56,000$ and the extra installation costs to use 16 digital cameras to get the displacement measurements of only $15 \%$ of the suspension part. One or two GPS receivers can be installed with the amount of $\$ 56,000$. However, it may be feasible to spend the same amount of money obtain the displacement measurements of $76 \%$ of the suspension part which probably significantly contributes to developing a more compelling mode shape model.

A thing is whether the required resolution of $5 \mathrm{~mm}$ is allowable. In the consideration that the maximum displacements of the Youngjong Bridge are approximately $35 \mathrm{~mm}$ which is relatively small compared to those of other super long-span bridges, it seems that the required resolution of $5 \mathrm{~mm}$ is not too rough to measure the displacement. Therefore, it seems feasible to use digital cameras to measure the displacements of a super long-span bridge.

\section{CONCLUSIONS}

From a case study with the EOS 5D Mark II camera from Cannon, it was found that digital cameras can provide a sufficient measuring width with a moderate resolution to measure the displacements of a super long-span bridge. This finding indicates the feasibility of digital cameras as the supplementary approach for the displacement measurements of a super long-span bridge. With the displacement measurements by digital cameras, a more compelling mode shape model will be developed.

There are several limitations in this study. While the feasibility of digital camera were investigated focusing on the measuring resolution, the measuring accuracy was out of the scope of this study. However, the measuring accuracy must be satisfied to employ digital cameras to measure the displacements of a super long-span bridge. A skew coefficient or distortion coefficients were also not dealt with in this study. These coefficients may not significantly affect the measuring resolution, but still need to be checked. These issues will be discussed in other studies later.

\section{ACKNOWLEDGMENTS}

This research was supported by a grant (code 10 Technology Innovation E05) from Construction Technology Innovation Program funded by Ministry of Land, Transportation and Maritime Affairs (MLTM) of Korean government.

\section{REFERENCES}

[1] Bae, I., and Ha, G., "Structural Health Monitoring of Long-Span Suspension Bridge Using GPS", In proceeding of 2008 KSCE Annual Conference, pp. 286-289, 2008.

[2] Jeong, Y., Kwark, J., Choi, E., Chin, W., and Lee, J., "Development of the Noncontact Vibrometer for the Monitoring System of Infrastructures", In proceeding of 2005 KSNVE Annual Spring Conference, pp. 563-566, 2005. 